\section{COMPLIANCE WITH A SMILE}

The Tracksense Pro is an efficient new decontamination validation process for autoclaves and sterilising devices.

Produced using the very latest technology, the new validation product and process consists of a pressure-temperature module and a dual temperature module. This complex data logging device meets industry requirements but is very simple to use. Clients are sent the validator, a member of staff places it in the autoclave and runs the cycle, the validator is returned in a freepost envelope and then a fully trained autoclave engineer assesses the data and sends the client all the required certification paperwork for their decontamination records.

The Tracksense Pro is quick, simple and significantly cheaper than other validation options. It enables practices to keep on top of regular testing and maintain consistent compliance with CQC regulations.

www.smilemaintenance.co.uk/ dental

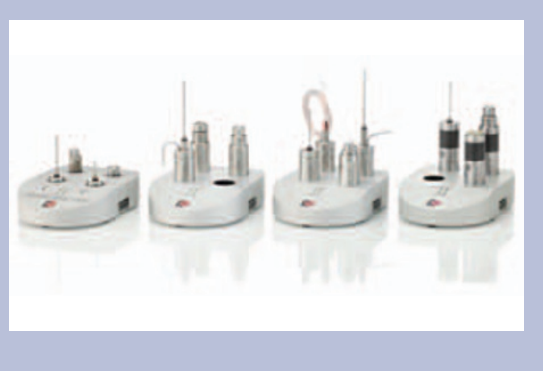

\section{WRIST MOVEMENT ONLY}

Physics Forceps are a revolutionary concept that enable dentists to extract teeth quicker, more efficiently, more predictably and with less trauma for the patient. They dramatically reduce the amount of time required to extract teeth and make the whole procedure much more comfortable and pleasant for both the operator and patient.

Physics Forceps have a revolutionary 'beak \&t bumper' design that enables dentists to extract teeth using wrist movement only - no more 'reaching for the apex'. Dramatically reducing the stress and strain experienced by patients, they also help preserve the buccal plate of bone, which is vital for the patient's long term dental health and success of future treatment options including dentures, bridges and implants.

To all intents and purposes Physics Forceps eliminate the need for surgical flaps, facilitate efficient extraction of practically every tooth (no matter what

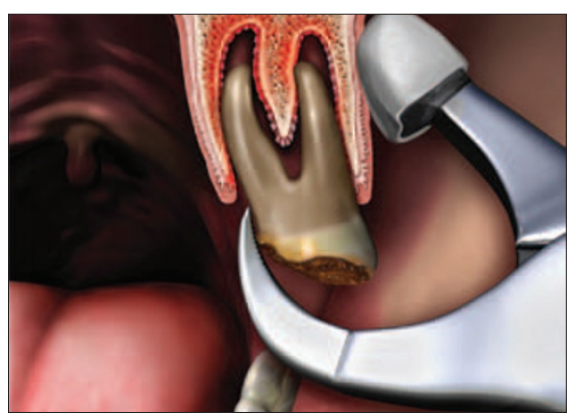

the condition) and achieve virtually atraumatic extractions in just a few minutes.

Physics Forceps are supplied with a comprehensive training DVD which explains the quite different, but very easy to learn, Physics extraction technique. They are also supplied with a 60 Day Money Back Guarantee (subject to restock charge) and a ten-year warranty following customer registration.

For further information call 01380 734990, visit www.generalmedical.co.uk oremailinfo@generalmedical.co.uk.

\section{A BROLLY WITH YOUR BRUSHES}

TePe Angle is the latest addition to the popular family of TePe Interdental Brushes.

TePe Angle was developed to improve access to all interdental spaces, particularly in difficult to reach areas. The angled head gives perfect access to posterior teeth without the need to bend the wire thus enhancing durability. The long and flat handle provides a stable, ergonomic grip and allows access both palatally and buccally.

TePe Angle is available in six colour coded sizes and all TePe interdental brushes have plastic coated wires for safety and come with a hygienic cover.

Order a minimum of five packs during March and April from your wholesaler and collect a stylish umbrella for free.

Visit www.molarltd.co.uk, email info@molarltd.co.uk or telephone 01934710022.

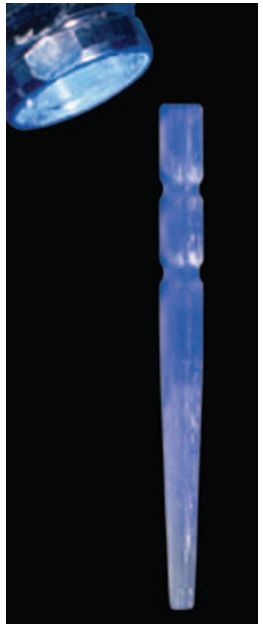

GLASS FIBRE REINFORCED COMPOSITE POST

\section{DMG UK's}

LuxaPost is a glass fibre reinforced composite post and forms the perfect partnership for LuxaCore Z-Dual composite core build up material.

LuxaPost has a low flexural modulus, which means its degree of elasticity and rigidity is virtually the same as dentine, thus minimising stresses within the post-cement-root combination and potential risk of root fractures.

Adhesive cementation ensures a secure and lasting bond while small retention grooves further improve mechanical retention. Its tapered form corresponds to that of the root and thus enables root friendly preparation, again reducing the risk of root fracture. Finally, its high transparency guarantees a perfect aesthetic appearance because it does not shine through the overlying restoration, unlike metal posts which frequently produce a greying effect.

LuxaPost is available in three sizes (1.25 mm, $1.375 \mathrm{~mm}$ and $1.5 \mathrm{~mm}$ ) with matching drills. The Intro Kit contains everything you need. Components are available in refill packs.

For further information contact your local dental dealer or DMG Dental Products (UK) Ltd on 01656 789401, email info@dmg-dental.co.uk or visit www.dmg-dental.com. 